\title{
Real-time Detection of Polymerization Reactions with Hyperpolarized Xenon at Low Magnetic Fields
}

Stefan Glöggler, Bernhard Blümich, and Stephan Appelt

Citation: AIP Conference Proceedings 1330, 101 (2011);

View online: https://doi.org/10.1063/1.3562243

View Table of Contents: http://aip.scitation.org/toc/apc/1330/1

Published by the American Institute of Physics 


\title{
Real-time Detection of Polymerization Reactions with Hyperpolarized Xenon at Low Magnetic Fields
}

\author{
Stefan Glöggler*, Bernhard Blümich*, Stephan Appelt ${ }^{\dagger *}$ \\ * Institute of Technical and Macromolecular Chemistry, RWTH Aachen University, Worringer Weg 1, 52074 Aachen, \\ Germany \\ ${ }^{\dagger}$ Central Institute for Electronics, Research Center Jülich, Wilhelm-Johnen-Straße, 52428 Juelich, Germany
}

\begin{abstract}
For process control it is desirable to develop simple devices for studying polymerization reactions in real-time and in-situ. We are demonstrating an approach using NMR at fields as low as $35 \mathrm{G}$ and hyperpolarized xenon, which allows us to observe polymerization reactions in real-time. The investigated reaction is a free radical polymerization with the initiator azobisisobutyronitrile (AIBN) and the monomer methyl methacrylate (MMA). AIBN and MMA are mixed together in a sample tube under noble gas atmosphere, and the reaction is started by irradiation with UV light (360 nm). As the reaction goes on, xenon NMR spectra are acquired. They show increasing line broadening and a variation of the chemical shift depending on the state of polymerization. This observation gives rise to the idea that a single-sided high resolution NMR sensor can be developed with which at least light induced polymerization reactions can be studied in-situ and in real-time.
\end{abstract}

Keywords: hyperpolarized xenon, polymerization, low magnetic field

PACS: $82.56 . \mathrm{Ub}, 36.20 . \mathrm{Fz}$

\section{INTRODUCTION}

Recently an emulsion polymerization reaction was investigated online with proton NMR at $0.5 \mathrm{~T}$ [1]. Due to the fact that averaging during the measuring process was necessary we were looking for a method to detect a polymerization reaction in real-time. In the past, hyperpolarized xenon has proven to be a powerful approach [2-4] in probing and differentiating between organic liquids at low magnetic fields. So we followed the approach to continuously introduce hyperpolarized xenon into the NMR sample as polymerization takes place in order to investigate polymerization reaction in real-time at low magnetic fields. Here we report the investigation of a lightinduced free radical bulk polymerization of methyl methacrylate initiated by azobisisobutyronitrile, as described in the literature [5], under continuous xenon gas flow investigated by ${ }^{129}$ xenon-NMR.

\section{EXPERIMENTAL SECTION}

For the bulk polymerization, $0.016 \mathrm{~g}$ of AIBN were added to $1 \mathrm{ml}$ of MMA, both purchased at Sigma-Aldrich, and placed inside a homebuilt open gap electromagnet which has a homogeneity of $1 \mathrm{ppm}$ without shims. The magnetic field was regulated to $3.5 \mathrm{mT}$ which corresponds to a frequency of $41 \mathrm{kHz}$ for ${ }^{129} \mathrm{Xe}$. Hyperpolarized xenon was produced by a homebuilt flow-polarizer and supplied through a $7 \mathrm{~m}$ long plastic tube into the sample cell. In order to start the reaction, AIBN was activated by the light of a LED (360 nm wavelength and $500 \mathrm{~mW}$ ) which was coupled to the sample cell inside the magnet by a waveguide. A schematic diagram of the NMR device and the light coupling is shown in Figure 1.

During the reaction, a $500 \mathrm{~cm}^{3} / \mathrm{min}$ flow of gas was supplied into the sample, which contained $1 \%$ hyperpolarized xenon and $99 \%$ inert gas. As the hyperpolarised xenon gas is constantly exchanged with the xenon in the polymer, it was possible to measure FID's at any given time in single-shot experiments.

Magnetic Resonance in Porous Media

AIP Conf. Proc. 1330, 101-104 (2011); doi: 10.1063/1.3562243

(C) 2011 American Institute of Physics 978-0-7354-0885-2/\$30.00 


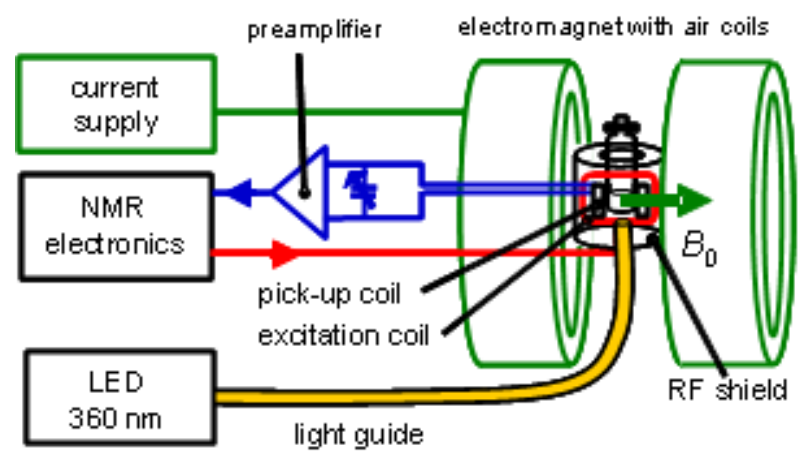

FIGURE 1. Open gap electromagnet with polymer sample and LED light coupled to the sample with a waveguide.

\section{RESULTS AND DISCUSSION}

Figure 2 shows two out of many FID signals and the corresponding xenon spectra acquired during light induced polymerization. In Fig. 2 (a), acquired after 5 minutes of irradiation, the signal shows an obvious beat which is not observable anymore after 120 minutes of irradiation. Fig. 2 (b) shows the corresponding spectra for both times. The xenon gas peaks have almost the same intensity and full width at half maximum (FWHM) whereas the intensity of the dissolved-phase peak has decreased down to a fifth of the initial value after 120 minutes of irradiation. The chemical shift difference between the xenon gas peak and the dissolved-phase peak has increased after 120 minutes from $169.0 \mathrm{ppm}$ to $177.9 \mathrm{ppm}$ and the FWHM has doubled from $0.009 \mathrm{~Hz}$ (2.2 ppm) to $0.18 \mathrm{~Hz}$ (4.4 ppm).

Observed exchange rates between xenon gas and the polymer solution are in the order of $1 \mathrm{~s}^{-1}$, which allows us to monitor the reaction steps every $10 \mathrm{~s}$. As the polymerization advances the solution will become heterogeneous consisting of more viscous parts with long chains and less viscous parts with short chains. Xenon, which is monitored in these experiments probes the less viscous parts. The high mobility of xenon in the less viscous parts leads to an insignificant dipolar broadening and thus to a narrow FWHM of $0.18 \mathrm{~Hz}$. In future, it is planned to shake the sample with ultrasonic inside the NMR probe, so that xenon can be dissolved in less than $1 \mathrm{~s}$.
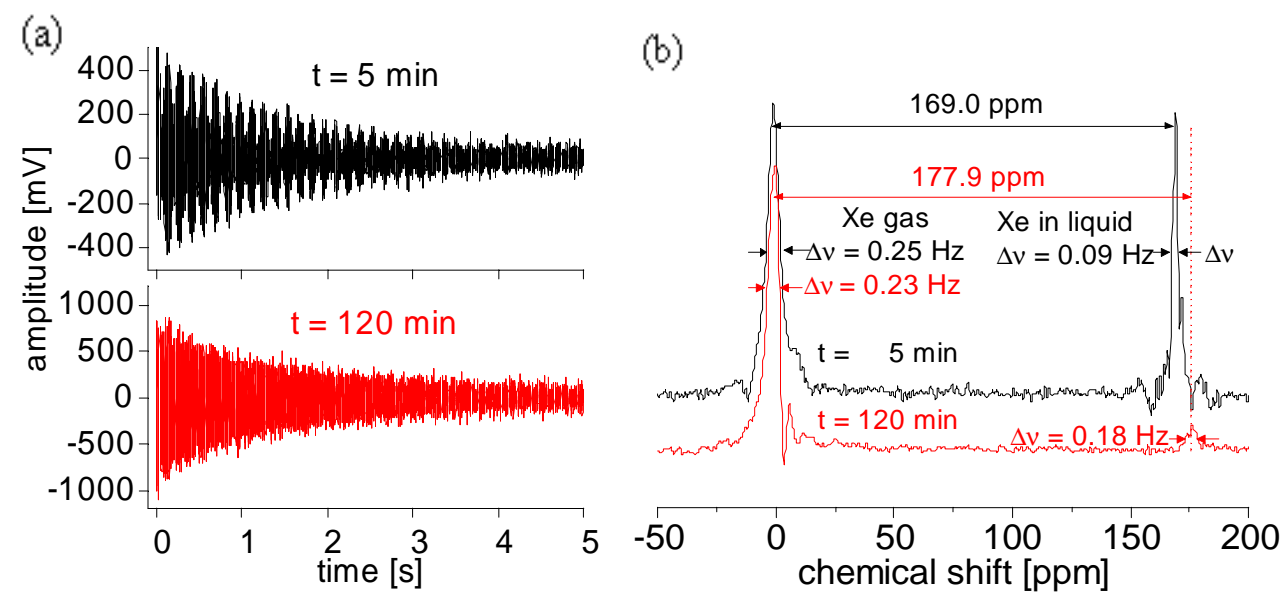

FIGURE 2. Xe-NMR of a MMA-monomer/polymer mixture. (a) free induction decays (FID) after 5 minutes and 120 minutes of irradiation with LED light. (b) Corresponding spectra. The spectral amplitudes are normalized to the Xe gas peak. Chemical shift and FWHM of the xenon peaks in the monomer/polymer solution increase during the reaction whereas the FWHM of the gas peak remains stable.

In Figure 3 (a) the spectra of the advancing polymerization reaction are presented while light was irradiated. It 
can clearly be seen that the chemical shift and FWHM increase as the signal intensity decreases. In higher magnetic fields one would observe two peaks: one for the monomer and one for the polymer which were not resolved at 3.5 $\mathrm{mT}$ because the chemical shift difference of those lines is about $2 \mathrm{ppm} \mathrm{[6]} \mathrm{and} \mathrm{the} \mathrm{most} \mathrm{narrow} \mathrm{line} \mathrm{width} \mathrm{that} \mathrm{was}$ recorded here is $2.2 \mathrm{ppm}$. Also in Fig. 3 (a) it is shown that there are fluctuations to the left and the right of the main peak which originate from fluctuations caused by the drift of the electromagnet and disturbances from the outside which affect the magnetic field because the magnet is not shielded.

In Fig. 3 (b) chemical shifts of dissolved xenon are compared from polymerization reactions, which are irradiated by light with $500 \mathrm{~mW}$ power and $50 \mathrm{~mW}$ power. In both reactions the change of chemical shift shows a linear behavior with time and the slope of the linear regression is larger for the monomer/polymer mixture irradiated with light of 500mW power. It is known [5] that for photo-initiated reactions the initiation rate is proportional to the quantum yield, the light intensity, the extinction coefficient and the concentration of the initiator. This means for the investigated reaction that more initiator is activated and polymer chains start propagating in a shorter time if the power of the light is higher. It is also known that the propagation rate of a radical polymerization shows first order behavior with respect to the monomer and polymer radical concentration; so it is possible to determine the reaction constant from the slope of the chemical shift changes.
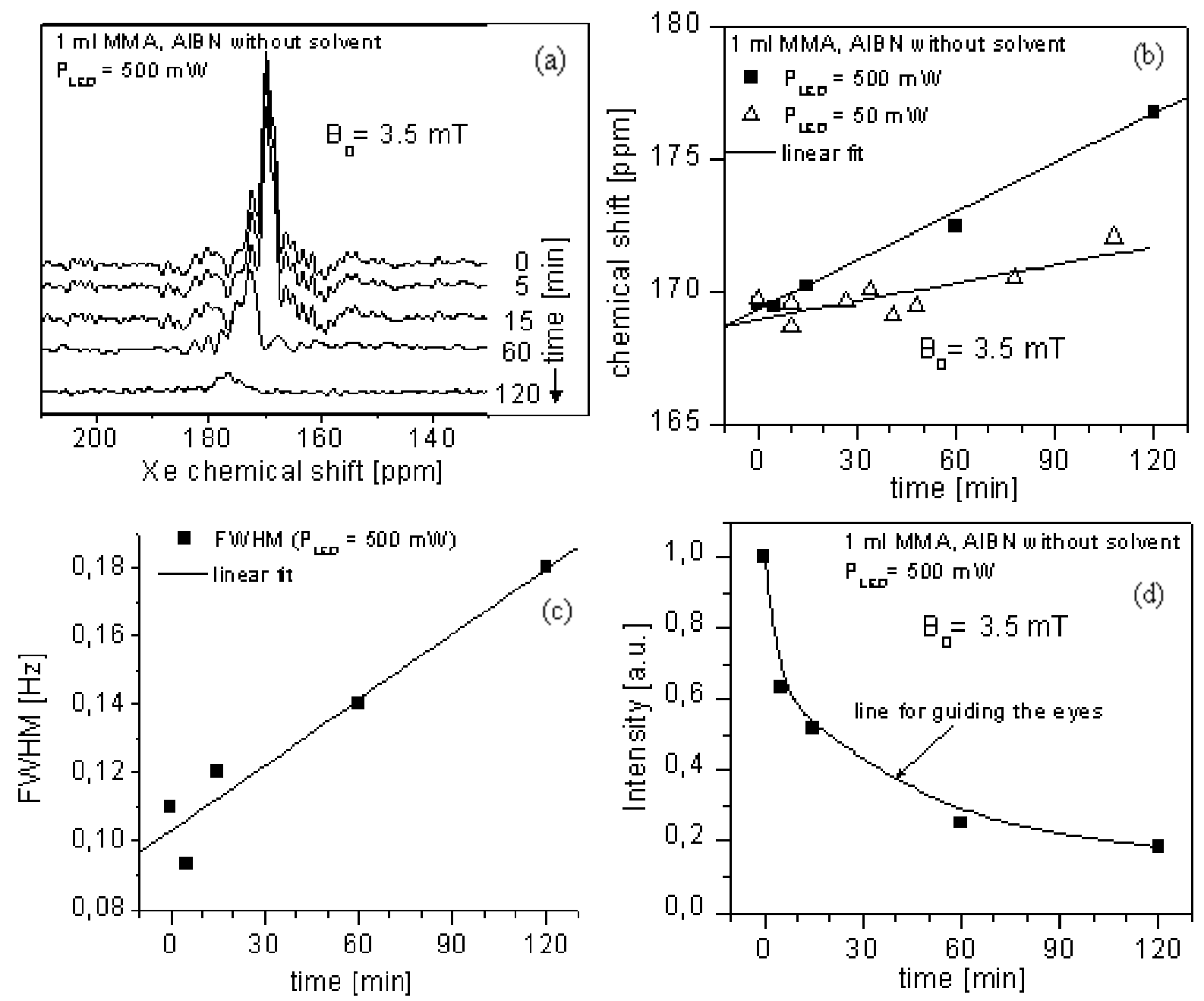

FIGURE 3. Polymerization of MMA with AIBN as initiator during irradiation with UV-light. (a) NMR spectrum of xenon dissolved in the sample as a function of time at $3.5 \mathrm{mT}$. (b) Chemical shift of the xenon peak in the monomer/polymer mixture at two different irradiation powers as a function of time. (c) FWHM of the dissolved xenon peak as a function of time. (d) Decrease of the xenon-peak intensity of the dissolved xenon as a function of time.

The FWHM values as a function of time as shown in Fig. 3 (c) also indicate the polymerization process. As the polymerization advances, the polymer becomes stiffer which means that the mobility of the chains decreases 
resulting in an increasing net xenon-proton dipolar coupling and the xenon peaks become broader [7]. Because of the fluctuations during the measurement it is almost impossible to determine the exact line width but the trend of broadening can be definitely observed.

Also the decrease of peak intensity, down to a fifth of the initial value (Fig. 3 (d)), is a measure of the polymerization process. It becomes harder for the xenon gas to penetrate the sample as the reaction advances because the polymer becomes stiffer. Therefore less xenon spins in the dissolved-phase are observed in the spectra with increasing polymerization time.

The last point we like to discuss are the low costs of the demonstrated method. Costs of xenon gas used for a continuous flow over 120 minutes are about 30 US-\$. In addition, it is possible to recycle the gas if a cold trap is incorporated into the system after the sample. Most residues in the used gas are trapped and the purified gas can be used over and over again.

\section{CONCLUSION}

We have demonstrated that it is possible to monitor light-induced polymerization reactions in real-time with simple NMR devices and low costs of hyperpolarized xenon at $3.5 \mathrm{mT}$. Also, it should be possible to determine reaction constants from the changes in chemical shift during the reaction and we have shown that chemical shifts, line widths and peak intensities are correlated to the degree of polymerization.

\section{ACKNOWLEDGMENTS}

The authors acknowledge generous support from K. Ziemons, J. Schmitz, G. D'Orsaneo, A. Schwaitzer, H. Glückler, U. Sieling and A. Gordji-Nejad from the Research Center Jülich for excellent technical assistance and helpful discussions.

\section{REFERENCES}

1. M. A. Vargas, M. Cudaj, K. Hailu, K. Sachsenheimer and G. Guthausen, Macromolecules, 43 (13), 5561-5568 (2010).

2. S. Appelt, F.W. Häsing, S. Baer-Lang, N. J. Shah, and B. Blümich, Chem. Phys. Lett. 348, 263 (2001).

3. S. Saxena, A. Wong-Foy, A. J. Moule, J. A. Seeley, R. McDermott, J. Clarke, and A. Pines, J. Am. Chem. Soc. 123, 8133 (2010)

4. A. Wong-Foy, S. Saxena, A. J. Moule', H.-M. L. Bitter, J. A. Seeley, R. McDermott, J.Clarke, and A. Pines, J. Magn. Reson. 157, 235 (2002).

5. J.M.G. Cowie, Polymers: Chemistry and Physics of modern materials $2^{\text {nd }}$ ed., CRC Press, Boca Raton, 1991.

6. M. Baias, "Science and History explored by NMR", Ph.D. Thesis, Shaker Verlag, Aachen, 2009.

7. A. P. M. Kentgens, H. A. van Boxtel, R.-J. Verweel, W. S. Veeman, Macromolecules 24, 3712-3714 (1991). 